\section{AB002. Lymph node dissection under minimal invasive surgery}

\section{René Horsleben Petersen}

Department of Cardiothoracic Surgery, Copenhagen University, Copenhagen, Denmark

Correspondence to: René Horsleben Petersen, MD, PhD. Department of Cardiothoracic Surgery, Copenhagen University, Rigshospitalet Blegdamsvej 9, Copenhagen 2100 Ø, Denmark.

Email: rene.horsleben.petersen@regionh.dk.

Abstract: A minimal invasive approach for lung cancer has become standard of care in many major thoracic centres around the world. In our institution at the Department of Thoracic Surgery at the University Hospital of Copenhagen, $90 \%$ of all lung cancer operations are performed with a minimal invasive approach. However implementation was slow in the beginning of the minimal invasive era. Thoracic surgeons were concerned about two major issues preventing implementation. Firstly, how to control major bleeding from the pulmonary artery? Secondly, the oncological efficacy of a minimal invasive approach was a concern. Many thoracic surgeons doubted that a systematic lymph node dissection under minimal invasive surgery could be performed with the same quality as in open surgery. Like in any other surgical approach, there is a considerable learning curve for learning the skills to perform a minimal invasive lobectomy. It is my experience from proctoring many thoracic surgeons around the world in video-assisted thoracoscopic surgery (VATS) lobectomy, that first step is to learn the techniques of performing the lobectomies. Then the technique of performing lymph node dissection has a separate learning curve to overcome. The key recommendation is to perform an "en bloc" resection with all lymphatic tissue and fatty tissue in one station. Keeping the capsule of the lymph intact decreases the risk of tumour cell spreading. Removing the lymph nodes this way makes it easier and more reliable for the pathologist to count the number of lymph nodes removed. The lymph node dissection is usually performed using monopolar electro coagulation (electro cautery hook) or using bipolar advanced energy devices such as Ligasure or Harmonic scalpel. The electro cautery hook is less costly and easy to use. However, there is a risk for injuring the pulmonary artery during dissection and the there is a considerable risk of lateral thermal spread up to $1 \mathrm{~cm}$ for the target area. This should be taken into consideration when dissecting near the recurrent nerve in the aorta-pulmonary window. Using advanced energy on the other hand is more expensive, however bipolar advanced energy devices can be used in close proximity to vascular structures and nerves with minimal risk of injury. Limiting bleeding by sealing minor vessels improves the overview of the dissection and thereby makes the dissection easier and safer. On the right side the lymph node dissection in the superior mediastinum is performed as an "en bloc" resection of the lymph nodes in station $2 \mathrm{R}$ and $4 \mathrm{R}$. The dissection begins inferior to the azygos vein along the pulmonary artery lifting the azygos vein up. The azygos vein can be divided, but in most cases this is not necessary. The smaller vessels from the superior vena cava (SVC) are sealed. Dissection continues along the SVC, the pericardium and trachea. Pleura over this area are divided and the dissection is continued to the top area near the subclavian artery. In the angle between the subclavian artery and trachea dissection should be performed carefully to avoid injuring the recurrent nerve. The specimen with lymph nodes and fatty tissue is removed via the utility port, which should be protected with a port protector to avoid seeding of tumour cells. The subcarinal area (station 7) is approached superior to the inferior vein. Dissection is continued until the left main bronchus is seen and $2 \mathrm{~cm}$ of the left main bronchus should be cleared for lymph nodes along with bronchus intermedius. Oesophagus can be retracted with the suction to open the space and facilitate dissection. It is recommended to divide the inferior ligament, when removing the paraoesophageal lymph nodes (station 8) and the lymph nodes in the lymph nodes in the inferior ligament (station 9). On the left side, lymph node dissection in the aorta-pulmonary window (stations 5 and 6) is initiated along the superior part of the aortic arch, identifying the vagal nerve. The recurrent nerve can easily be localized, when following the vagal nerve to the inferior part of the aortic arch. Once the recurrent nerve is identified, the risk of injury and traction is less. The majority of injuries to recurrent nerve during lymph node dissection is due to traction and causes neuropraxia. Fortunately this type of injury is temporary, however patients are often hoarse for several months. After the recurrent nerve is localized, dissection continues along the phrenic nerve and pleura are opened. The dissection continues along the main pulmonary artery and all the 
lymph nodes and fatty tissue can be removed via the utility port and a port protector like the right side. The lymph nodes in stations 7, 8 and 9 can removed similar to removal on the right side, however the dissection is deeper in the subcarinal area on the left side and retraction with 2 or more instruments facilitates the dissection.

Keywords: Lymph node dissection; video-assisted thoracoscopic surgery lobectomy (VATS lobectomy); minimal invasive thoracic surgery

doi: $10.21037 /$ shc.2019.AB002

Cite this abstract as: Petersen RH. Lymph node dissection under minimal invasive surgery. Shanghai Chest 2019;3:AB002. 